\title{
Spatio-Temporal Games Beyond One Dimension
}

\author{
SOHEIL BEHNEZHAD, University of Maryland \\ MAHSA DERAKHSHAN, University of Maryland \\ MOHAMMADTAGHI HAJIAGHAYI, University of Maryland \\ SAEED SEDDIGHIN, University of Maryland
}

Protecting valuable targets from an adversary is an ever-important international concern with far-reaching applications in wildlife protection, border protection, counter-terrorism, protection of ships from piracy, etc As a successful recent approach, security games cast these issues as two-player games between a defender and an attacker. The defender decides on how to allocate the available resources to protect targets against the attacker who strives to inflict damage on them. The main question of interest here is equilibrium computation

Our focus in this paper is on spatio-temporal security games. However, inspired by the paper of Xu [EC'16], we start with a general model of security games and show that any approximation (of any factor) for the defender's best response (DBR) problem leads to an approximation of the same factor for the actual game.

In most applications of security games, the targets are mobile. This leads to a well-studied class of succinct games, namely spatio-temporal security games, that is played in space and time. In such games, the defender has to specify a time-dependent patrolling strategy over a spatial domain to protect a set of moving targets. We give a generalized model of prior spatio-temporal security games that is played on a base graph $G$. That is, the patrols can be placed on the vertices of $G$ and move along its edges over time. This unifies and generalizes prior spatio-temporal models that only consider specific spatial domains such as lines or grids. Graphs can further model many other domains of practical interest such as roads, internal maps of buildings, etc.

Finding an optimal defender strategy becomes NP-hard on general graphs. To overcome this, we give an LP relaxation of the DBR problem and devise a rounding technique to obtain an almost optimal integral solution. More precisely, we show that one can achieve a $(1-\epsilon)$-approximation in polynomial time if we allow the defender to use $\lceil\ln (1 / \epsilon)\rceil$ times more patrols. We later show that this result is in some sense the best possible polynomial time algorithm (unless $\mathrm{P}=\mathrm{NP}$ ). Furthermore, we show that by using a novel dependent rounding technique, the same LP relaxation gives an optimal solution for specific domains of interest, such as one-dimensional spaces. This result simplifies and improves upon the prior algorithm of Behnezhad et al. [EC'17] on several aspects and can be generalized to other graphs of interest such as cycles.

Lastly, we note that most prior algorithms for security games assume that the attacker attacks only once and become intractable for a super-constant number of attacks. Our algorithms are fully polynomial in the input size and work for any given number of attacks.

\section{CCS Concepts: • Theory of computation $\rightarrow$ Algorithmic game theory and mechanism design;}

Additional Key Words and Phrases: security games, Nash equilibrium, ellipsoid method, approximation

Supported in part by NSF CAREER award CCF-1053605, NSF BIGDATA grant IIS-1546108, NSF AF:Medium grant CCF1161365, DARPA GRAPHS/AFOSR grant FA9550-12-1-0423, and another DARPA SIMPLEX grant

Authors' addresses: Soheil Behnezhad, University of Maryland, soheil@cs.umd.edu; Mahsa Derakhshan, University of Maryland, mahsa@cs.umd.edu; MohammadTaghi Hajiaghayi, University of Maryland, hajiagha@cs.umd.edu; Saeed Seddighin, University of Maryland, saeedreza.seddighin@gmail.com.

Permission to make digital or hard copies of all or part of this work for personal or classroom use is granted without fee provided that copies are not made or distributed for profit or commercial advantage and that copies bear this notice and the full citation on the first page. Copyrights for components of this work owned by others than ACM must be honored. Abstracting with credit is permitted. To copy otherwise, or republish, to post on servers or to redistribute to lists, requires prior specific permission and/or a fee. Request permissions from permissions@acm.org.

(C) 2018 Association for Computing Machinery.

ACM EC'18, June 18-22, 2018, Ithaca, NY, USA. ACM ISBN 978-1-4503-5829-3/18/06 .. \$15.00

https://doi.org/10.1145/3219166.3219226 


\section{INTRODUCTION}

Protecting a set of valuable targets from an adversary is a critical international concern with far-reaching applications in wildlife protection, border protection, counter-terrorism, protection of ships from piracy, etc. As a successful recent approach, security games cast these issues as twoplayer games between a defender and an attacker. The defender has to decide on how to allocate her resources to provide the best protection against the attacker who strives to inflict damage on the targets.

The most common solution concept that is adopted for security games in the literature is a strong Stackelberg equilibrium. The defender commits to a particular randomized allocation (e.g., a randomized patrolling schedule) whereas the attacker attacks at will. The defender's strategy is assumed to be revealed to the attacker due to the ability of the attacker in using surveillance to learn about the defender's strategy. The attacker then best-responds to this strategy by attacking a target that maximizes the expected damage. Therefore, the defender's goal is to play her optimal maximin strategy.

This approach has inspired a plethora of studies and research activities, including several awards and nominations (see Section 1.2 for an overview of related work). Remarkably, it has also been adopted in real-world scenarios such as airport checkpoints, US air marshals, urban transit systems and wildlife protection; many of these were recognized with commendations and awards. Security games also have other potential applications in protecting ships from piracy or protecting aid convoys in unstable regions.

In most applications of security games, the targets are mobile. This leads to a well-studied class of security games that is played in space and time $[3,8,19]$. In spatio-temporal security games, the defender has to specify a time-dependent patrolling strategy over a spatial domain to protect a set of moving targets. Figure 1 illustrates a spatio-temporal security game that is played on a discretized one-dimensional space. Time and space are respectively illustrated on $\mathrm{x}$ - and $\mathrm{y}$-axis. The paths denote the trajectory of two patrols and the stars denote targets. The hatched area around the patrol paths denote their protection area.

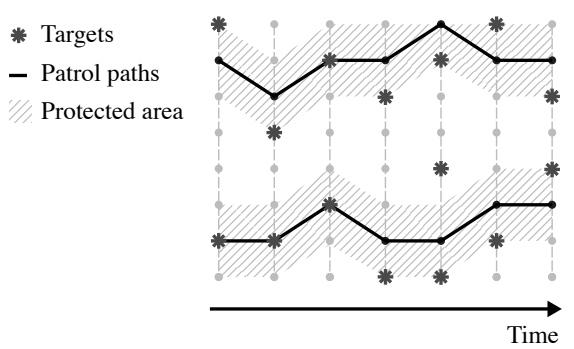

Fig. 1. A game instance on a path.

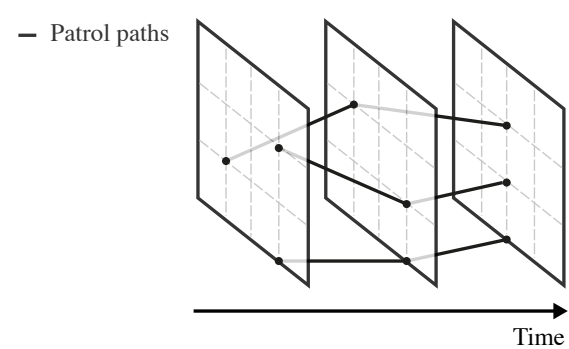

Fig. 2. Patrols moving on a grid.

The number of pure strategies of the defender in spatio-temporal security games is often exponentially large. This, combined with the overlapping protection ranges of the patrols, makes it notoriously difficult to find an optimal strategy in polynomial time. Even the seemingly easy case of discretized one-dimensional domains is quite a challenge and existence of a polynomial time algorithm remained open until the recent algorithm of [3] that solves it in polynomial time.

For some real-world applications of spatio-temporal security games such as border protection, the spatial domain is indeed one-dimensional. However, for many cases, the space is much more complicated. In wildlife protection, for instance, the targets (animals) can move in a rather complicated 
two-dimensional area. Ignoring complex natural objects such as hills or rivers, such environments can be modeled by grids [9]. However, there are many different instances that require even more complicated spatial domains.

In this paper, we consider spatio-temporal security games on graphs. That is, we assume that the underlying spatial domain is represented by an undirected graph $G$ where the patrols can be placed on the vertices of $G$ and move along its edges. This unifies and generalizes the aforementioned prior models. For instance, the graph for one-dimensional domains is simply a path ${ }^{1}$ and discretized two-dimensional spaces can be modeled by grids (Figure 2). Graphs allow us to model even more complicated spatial domains such as roads, internal map of buildings, etc.

\subsection{Our Results \& Techniques}

Most variants of security games can be shown to be bilinear games (see Section 3). That is, for any fixed strategy of the attacker, the defender's utility can be computed by a linear function (and vice versa). This allows us to compute the optimal minimax strategy of the defender via a linear program with roughly the same number of variables as the number of strategies of the defender and the same number of constraints as the number of strategies of the attacker (see LP 1).

The main challenge, however, lies in the fact that the number of strategies of both the defender and the attacker is exponentially large. That is, the defender can put her $K$ patrols in any subset of the vertices at the first time point and move them over time. On the other hand, the attacker can attack any subset of size up to $Q$ of the targets.

The huge number of strategies of the players leads to having an LP with exponentially many variables and exponentially many constraints which cannot be solved in polynomial time. The overall approach is to reduce the number of variables to a polynomial and then use the ellipsoid method to handle the exponential number of constraints. For this, we first take dual of LP $1-$ given as LP 2 - which corresponds to the attacker's maximin strategy. LP 2 also has exponentially many variables and constraints, however, the difference is that the variables of LP 2 correspond to the attacker's pure strategies, whereas, the variables of LP 1 correspond to the defender's. We show that, fortunately, the inherent symmetry in attacker's strategies can be used to obtain an equivalent LP - given as LP 3 - with only polynomially many variables.

LP 3 can be solved using the ellipsoid method if we have access to a polynomial time separating oracle. The separating oracle is, in fact, equivalent to the defender's best response (DBR) problem which is the following combinatorial problem: Given a set of weighted targets over time, what is the pure strategy of the defender that covers the maximum weight? (See Definition 3 for the formal statement.) Therefore the complexity of solving spatio-temporal security games is linked directly to the complexity of this combinatorial problem. Indeed the main body of work in this work is devoted to solving (or approximating) the defender's best response problem for different variants of the game.

Using the oracle-based approach is nothing new and it has been used in the literature to find optimal strategies of certain types of succinct games, including security games [1, 10, 11, 13, 18, 19] However, the DBR problem is not necessarily easier to solve than the actual game. In fact, the recent paper of $\mathrm{Xu}$ [18] shows that any exact solution for the actual game leads to an exact solution for the DBR problem. Therefore, unsurprisingly, there had been many instances where the actual game is solved directly without any attempts to solve the DBR problem (e.g. see [3]).

\footnotetext{
${ }^{1}$ In paths the speed limit of patrols is 1, i.e., they can only move to an adjacent location after each time point. To generalize this to an arbitrary speed limit $\Delta$ that is considered in prior work, the graph would be a pseudo-path, where each vertex is connected to $\Delta$ nearby vertices.
} 
The DBR problem turns out to be NP-hard for many natural variants of spatio-temporal security games. Therefore, unless $\mathrm{P}=\mathrm{NP}$, we can only solve it approximately in polynomial time. An approximate solution might relax the number of patrols that are used or the fraction of the optimal solution that it achieves. Define a solution to be an $(\alpha, \beta)$-approximation if it achieves an $\alpha$ fraction of the optimal solution by using up to at most $\beta$ times more patrols (i.e., $\alpha \leq 1$ and $\beta \geq 1$ ). A natural question is whether an approximate DBR solution leads to an approximation of the actual game. Our first result answers this affirmatively.

Result 1. (Formally as Theorem 6). Any $(\alpha, \beta)$-approximation of the DBR problem leads to an $(\alpha, \beta)$-approximation of the actual game.

Using the known low-regret learning techniques, one can obtain an $\epsilon$-minimax equilibrium by having access to an additive FPTAS of DBR (see e.g., [10]). However, Result 1 is essentially different in that it preserves the multiplicative approx factors $\alpha$ and $\beta$. This generalization is essential for our contributions since the DBR does not admit any additive FPTAS in the general case (see Section 7). Having Result 1, we then focus on solving the DBR problem for different variants of spatio-temporal security games.

For spatio-temporal security games, the DBR problem is as follows: given $N$ targets that move over time, find a deterministic (i.e., pure) scheduling for $K$ homogeneous patrols that together maximize the total weight of protected targets. What complicates computation of the optimal scheduling is the existence of protection range around the patrols and the fact that double coverage has no benefit. To illustrate the computational hardness of the DBR problem, consider a game instance that is played only in one time unit (i.e., the targets and patrols do not move) and when there is a target of weight 1 on each vertex. Also assume that the protection range of each patrol is 1 (i.e., all targets at distance at most 1 are protected). If one can cover all the targets by $K$ patrols, then the dominating set of the base graph is at most $K$. A problem that is not only NP-hard, but also Log-APX-complete [7]. Also recall that the original DBR problem is much more complicated than this example; patrols and targets may move over time and the targets are not homogeneous.

Generally, to solve the DBR problem, we focus on an LP relaxation (formally given as LP 4) of the problem where we allow the patrols to move fractionally. That is, instead of integral paths, we assume that any flow of size one is a valid scheduling for a patrol. Note that the LP that computes the optimal fractional solution has to incorporate other constraints to prevent double coverage. Therefore, the standard integrality of network flow is not necessarily the case for our problem. After all, the problem is NP-hard in the general case, therefore, the integrality gap of this relaxation cannot be one. As such, the main technicality of our work is centered around different rounding techniques for the LP solution.

We first show that for general graphs, there exists a rounding scheme that achieves an almost optimal solution by using at most $\lceil\ln (1 / \epsilon)\rceil$ times more patrols. To obtain this, we first decompose the fractional solution into $K$ flows of size 1 , then pick $\lceil\ln (1 / \epsilon)\rceil$ paths independently (but not uniformly) at random from each of these flows. We later show that, at least for specific values of $\epsilon$, this bound is essentially tight (see Theorem 18).

Result 2. (Formally as Theorem 15). For any $\epsilon>0$, one can, in expectation, find in polynomial time, a $\left(1-\epsilon,\left\lceil\ln \frac{1}{\epsilon}\right\rceil\right)$-approximation of the DBR problem, and therefore a $\left(1-\epsilon,\left\lceil\ln \frac{1}{\epsilon}\right\rceil\right)$ approximation of the actual game, on general graphs.

The inapproximability results use, in an essential way, the generality of the underlying graph. However, in practice, we may be dealing with very specific graphs. For example, an important class 
of spatio-temporal security games are played in discretized one-dimensional (Figure 1) domains $[3,8,9,19]$ where the base graph would be a line. Therefore the question is: can we obtain any better rounding scheme when we have certain assumptions about the input graph? We show that, perhaps surprisingly, the integrality gap of LP 4 is indeed one for the one-dimensional case. Obtaining the following result.

Result 3. (Formally as Theorem 12). One can find in polynomial time, the optimal solution to the DBR problem, and therefore the actual game, for discretized one-dimensional spaces (i.e., lines or pseudo-lines).

To prove Result 3 , we first pick a mass variable $\sigma \in[0,1]$ uniformly at random; then construct each of the patrol paths according to $\sigma$. Roughly speaking, the main role of $\sigma$ is that, unlike the rounding for general graphs where the patrol paths are constructed independently from each other, it correlates how different patrols are rounded in a way that, we argue, will minimize doublecoverage. We then show that this procedure, in expectation, achieves the same coverage as the fractional solution. Recall that any integral solution is also a valid fractional solution, thus, no rounded solution can achieve a better coverage. This implies that in order to achieve the same expected coverage, for every choice of $\sigma$, we indeed achieve the same coverage as the fractional solution.

Result 3 improves the recent polynomial time algorithm of [3] on several aspects. First, [3] assume that the attacker attacks only once and their algorithm becomes intractable for even two attacks, whereas, our solution works for any given number of attacks. Second, we show that our solution can be generalized to many related graphs of interest, such as cycles, where it is arguably impossible to generalize the approach of [3] to obtain exact solutions. Third, our solution is significantly simpler than that of [3]. We also note that a problem similar to the DBR problem was considered in [19] for which the authors give a $(1-1 / e, 1)$-approximation. Combining this approximation with Result 1 , one can achieve a $(1-1 / e, 1)$-approximation of the actual game. Result 3 improves this by obtaining the exact solution.

\subsection{Related Work}

Security games have attracted a plethora of studies in the past decade, see the book [17] as well as more recent work, e.g. [3, 4, 8, 14, 18, 19]. Remarkably, this research has concerned both theory and practice; real-world deployments include: Canine-patrol and vehicle-checkpoints scheduling in Los Angeles airport (LAX) [15], US Coast Guard patrol boats [8], airport passenger screening by US Transportation Security Administration [5], scheduling flights for air marshals by US Federal Air Marshal Service [12], fare inspection in Los Angeles transit system [20], and wildlife protection in Malaysia [9].

Security game papers that are most relevant to this work address moving targets and patrols $[3,4,8,19]$ (as previously discussed). Other work concerned solving security games that are not (necessarily) spatio-temporal, e.g. [6,13,18].

Our work, in a broader context, is also related to Stackelberg games and equilibrium computation in succinct games. While it is known to be PPAD-hard in general, polynomial time algorithms exist for many natural variants. For background, see a survey [16]; or more recent papers in this context $[1,2,10]$.

\subsection{Paper Organization}

In Section 2 we describe the model. In Section 3 we cover required game theory basics and describe, in Section 4, the overall oracle-based approach. In Section 5 we show how to solve (or approximate) 
the defender's best response problem for general graphs and specific domains (specifically onedimensional domains) and in Section 6 show why this leads to an approximation of the actual game. Finally, in Section 7 we cover the hardness results.

\section{THE MODEL}

Security games are two-player games which are played between a defender and an attacker. In this paper, we focus on spatio-temporal security games where the goal of the defender is to protect a set of moving targets (e.g., ferries carrying passengers) from an attacker by choosing a time-dependent patrolling strategy (Figure 2 and Figure 1).

Environment. The game is played on a given graph $G=(V, E)$ where the defender's patrols and the targets can be positioned on the vertices of $G$. Furthermore, the temporal domain is assumed to be discretized into $T$ time points. We consider the full-information case where both the defender and the attacker have complete knowledge about the targets' movements a priori. ${ }^{2}$ The defender has $K$ homogeneous patrols that can move, in each time step, to any adjacent vertex. However, we assume that the state of targets in one time point does not give us any information about them at other time points (e.g., the targets are not restricted, in contrast to the patrols, to move only to adjacent vertices in one time step). ${ }^{3}$ In fact, from now on, we treat targets of different time points as $N$ totally different objects (i.e., $N=K \cdot N^{\prime}$ where $N^{\prime}$ denotes the number of targets in each time point). For any $i \in[N]$ we respectively denote by $t_{i} \in[T]$ and $\ell_{i} \in V$ the time and location of the $i$ th target.

Strategies. As it was already mentioned, the defender has $K$ homogenous patrols that after each time step can either move to an adjacent vertex or remain at their current location. Moreover, the patrols have a protection range $R$ within which any target is protected. More precisely, a patrol that is located at vertex $v$ protects any target in location $u$ as long as $\operatorname{dist}_{G}(v, u) \leq R$. A patrol path, which denotes the trajectory of a patrol, is a list $\left(v_{1}, v_{2}, \ldots, v_{T}\right)$ of size $T$ of vertices in $G$ where for any $t \in[T-1]$, either $v_{t+1}=v_{t}$ or $v_{t+1}$ is a neighbor of $v_{t}$ in $G$. A pure strategy of the defender is a set of $K$ patrol paths. A pure strategy of the attacker, on the other hand, is a subset $S_{A} \subseteq[N]$ of size at most $Q$ of the targets to attack.

Utilities. Associated to each target $i \in[N]$ is a weight $w_{i} \in \mathbb{R}_{+}$and a cost $c_{i} \in \mathbb{R}_{+}$. For any $i \in[N]$, we denote the partial utility of the defender (resp. attacker) on target $i$ by $\mathrm{U}_{i}^{\mathrm{D}}\left(\right.$ resp. $\left.\mathrm{U}_{i}^{\mathrm{A}}\right)$ and define it as follows.

$\mathrm{U}_{i}^{\mathrm{D}}=\left\{\begin{array}{ll}w_{i} & \text { if } i \text { is not attacked, } \\ w_{i}-c_{i} & \text { if } i \text { is attacked but protected, } \\ 0 & \text { if } i \text { is attacked and unprotected. }\end{array} \quad \mathrm{U}_{i}^{\mathrm{A}}= \begin{cases}0 & \text { if } i \text { is not attacked, } \\ c_{i} & \text { if } i \text { is attacked but protected, } \\ w_{i} & \text { if } i \text { is attacked and unprotected. }\end{cases}\right.$

The overall utility of the defender (resp. attacker), which we denote by $\mathrm{U}^{\mathrm{D}}$ (resp. $\mathrm{U}^{\mathrm{A}}$ ) is simply sum of utilities on all targets, i.e.,

$$
\mathrm{U}^{\mathrm{D}}=\sum_{i \in[N]} \mathrm{U}_{i}^{\mathrm{D}}, \quad \mathrm{U}^{\mathrm{A}}=\sum_{i \in[N]} \mathrm{U}_{i}^{\mathrm{A}} .
$$

Note that the game is constant sum with $\Gamma:=\sum_{i=1}^{N} w_{i}$ being the sum of utilities. We assume that $c_{i}<w_{i}$ for any target $i$; meaning that protecting a target is strictly beneficial to the defender than not protecting it. Intuitively, for patrolling ferries, one can think of $w_{i}$ as the number of passengers

\footnotetext{
${ }^{2}$ We remark that the stochastic case, where the players are only given the distribution of target locations can be reduced to the full-information case.

${ }^{3}$ The independence of targets of different time points is a standard assumption in the literature $[3,8,19]$. We also note that all of our hardness results in Section 7 work for the unit time case when $T=1$; meaning that it is essentially impossible to have any form of a time-dependent assumption on targets that makes the game solvable in polynomial time.
} 
in ferry $i$. All of the passengers get hurt if the attacker attacks it and the defender does not protect it; however, only $c_{i}$ passengers get hurt if the attacker attacks the ferry but the defender protects it. The defender's overall utility is the number of unhurt passengers and the attacker's overall utility is the number of passengers that get hurt. Therefore, sum of utilities is always the total number of passengers in the ferries.

The input. Any instance of the game can be uniquely represented by $G=(V, E), T, K, R, Q$, and the weights and costs of targets. The total input size, therefore, is of $\Omega(|V|+|E|+N+\log (K \cdot R \cdot Q))$ bits. All the algorithms that we give are polynomial time in the input size.

\section{GAME THEORY BACKGROUND}

Bilinear games. Consider a two-player constant-sum game $\mathcal{G}$ with $\Gamma$ denoting the sum of utilities and polytopes $\mathcal{S}_{1}$ and $\mathcal{S}_{2}$ denoting the players' strategy spaces. As in the literature, we call $\mathcal{G}$ a bilinear game if there exists a utility matrix $M$ such that for any strategy profile $(\mathbf{x}, \mathbf{y}) \in \mathcal{S}_{1} \times \mathcal{S}_{2}$, the first player gets utility $\mathbf{x}^{T} M \mathbf{y}$ (implying that the second player gets utility $\Gamma-\mathbf{x}^{T} M \mathbf{y}$ ).

Our model is not bilinear. However, the strategy spaces can be reformulated in a compact way that transforms the game into a payoff-equivalent bilinear game. To do so, let us represent each pure strategy of the defender by a vector $\left(x_{1}, \ldots, x_{N}\right)^{T}$ where $x_{i}=1$ if target $i$ is protected in the strategy and $x_{i}=0$ otherwise. Note that any valid strategy maps to a point in $\{0,1\}^{N}$ but the reverse is not always true, i.e., a point in $\{0,1\}^{N}$ does not necessarily represent a valid strategy. We denote by $\mathcal{S}^{D}$ the set of pure strategies of the defender in the new space. Furthermore, we represent each pure strategy of the attacker by a vector $\left(y_{1}, \ldots, y_{N}\right)^{T}$ in $\{0,1\}^{N}$ where $y_{i}=1$ if the attacker attacks target $i$ and $y_{i}=0$ otherwise. Similarly, we denote by $\mathcal{S}^{A}$ the set of all valid strategies of the attacker in the new space.

Mixed strategies are basically linear combinations of pure strategies. Therefore, in the new space, each mixed strategy of the defender and the attacker can be represented by a vector of size $N$, where each entry is a (possibly fractional) number in $[0,1]$. The $i$ th entry of any strategy of the defender denotes the marginal coverage probability of target $i$. Similarly, the $i$ th entry of any strategy of the attacker denotes the marginal attacking probability of target $i$.

By representing strategies in the marginal probability spaces, spatio-temporal security games become bilinear. If the defender and the attacker respectively play pure strategies $\mathbf{x} \in \mathcal{S}^{D}$ and $\mathrm{y} \in \mathcal{S}^{A}$, the utilities of the defender and the attacker respectively become

$$
\mathrm{U}^{D}(\mathbf{x}, \mathbf{y}):=\sum_{i \in[N]}\left(w_{i}-c_{i}\right) x_{i} y_{i}-w_{i} y_{i}+w_{i}, \quad \quad \mathrm{U}^{A}(\mathbf{x}, \mathbf{y}):=\sum_{i \in[N]}\left(c_{i}-w_{i}\right) x_{i} y_{i}+w_{i} y_{i} .
$$

Note that this is indeed a bilinear function of a slightly different form of $\mathbf{x}^{T} A \mathbf{y}+B \mathbf{y}+c$. The following lemma shows that utilities can essentially be computed via the same function for mixed strategies too.

Lemma 1. Given a mixed strategy $\mathbf{x}^{\prime}$ of the defender and a mixed strategy $\mathbf{y}^{\prime}$ of the attacker, the expected utility of the defender can be computed via the following function where $x_{i}^{\prime}$ and $y_{i}^{\prime}$ respectively denote the marginal coverage and marginal attacking probabilities for target $i$.

$$
U^{D}\left(\mathbf{x}^{\prime}, \mathbf{y}^{\prime}\right):=\sum_{i \in[N]}\left(w_{i}-c_{i}\right) x_{i}^{\prime} y_{i}^{\prime}-w_{i} y_{i}^{\prime}+w_{i}, \quad U^{A}\left(\mathbf{x}^{\prime}, \mathbf{y}^{\prime}\right):=\sum_{i \in[N]}\left(c_{i}-w_{i}\right) x_{i}^{\prime} y_{i}^{\prime}+w_{i} y_{i}^{\prime}
$$

The proof of Lemma 1 is included in the full-version of the paper.

Solution concepts. Many different solution concepts are of interest for security games. For example, a strong Stackelberg Equilibrium captures an equilibrium scenario where the leader (the defender) 
commits to a mixed strategy and the follower (the attacker) best-responds to this mixed-strategy. Other well-known solution concepts, such as the Nash equilibrium or minimax solutions have also been considered. The celebrated minimax theorem of von Neumann, states that all these solution concepts are equivalent to one another for constant-sum games. Hence, throughout the paper, we seek to compute only a maximin solution. That is, our goal is to find a strategy profile $(\mathbf{x}, \mathbf{y})$ where,

$$
\mathbf{x}=\underset{\mathbf{x}^{\prime}}{\arg \max }\left[\min _{\mathbf{y}^{\prime}}\left(\mathrm{U}^{D}\left(\mathbf{x}^{\prime}, \mathbf{y}^{\prime}\right)\right)\right], \quad \text { and } \quad \mathbf{y}=\underset{\mathbf{y}^{\prime}}{\arg \max }\left[\min _{\mathbf{x}^{\prime}}\left(\mathrm{U}^{A}\left(\mathbf{x}^{\prime}, \mathbf{y}^{\prime}\right)\right)\right] .
$$

Since it becomes computationally difficult to find the exact maximin strategies in some cases, we are also interested in finding approximate maximin strategies. A strategy $\mathbf{x}$ is an $\alpha$-approximate minimax strategy for the defender if it guarantees receiving an $\alpha$ fraction of the maximin value, or more precisely,

$$
\min _{\mathbf{y}^{\prime}}\left[\mathrm{U}^{D}\left(\mathbf{x}, \mathbf{y}^{\prime}\right)\right] \geq \alpha \cdot \max _{\mathbf{x}^{\prime}}\left(\min _{\mathbf{y}^{\prime}}\left[\mathrm{U}^{D}\left(\mathbf{x}^{\prime}, \mathbf{y}^{\prime}\right)\right]\right) \cdot{ }^{4}
$$

\section{A GENERAL APPROACH FOR SOLVING SECURITY GAMES}

Maximin strategies can be efficiently computed for bilinear games using a simple linear program if the players have polynomially many pure strategies. Unfortunately, the number of pure strategies of the defender in our game is exponentially large. Nevertheless, since our solutions essentially build upon this linear program, we start by stating defender's minimax linear program as LP $1 .{ }^{5}$

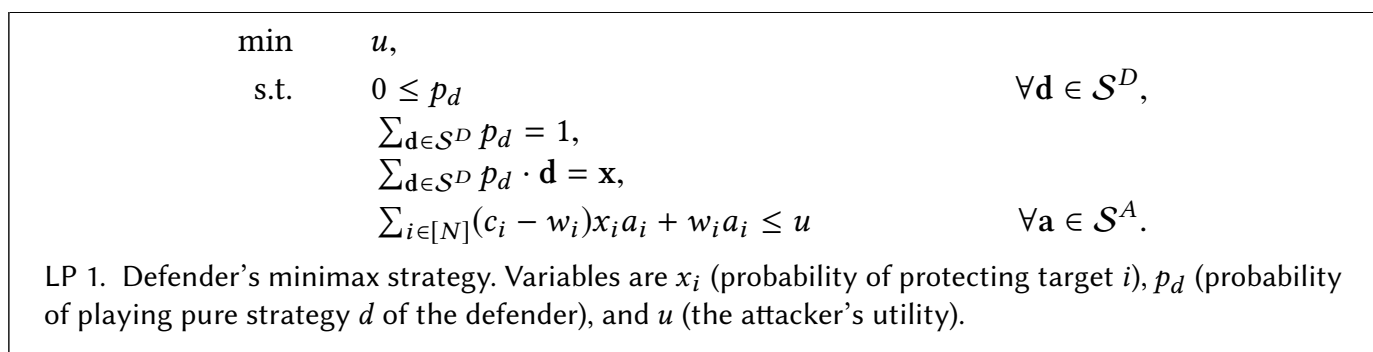

LP 1 has exponentially many variables and constraints, thus, we cannot directly solve it in polynomial time. Our goal is to convert it to another LP with polynomially many variables (but still with exponentially many constraints) and use ellipsoid method to solve it. To do this, consider

\begin{tabular}{|c|c|c|}
\hline $\begin{array}{c}\max \\
\text { s.t. }\end{array}$ & 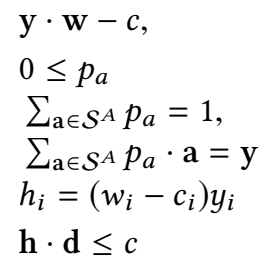 & $\begin{array}{l}\forall \mathbf{a} \in \mathcal{S}^{A}, \\
\forall i \in[N], \\
\forall \mathbf{d} \in \mathcal{S}^{D} .\end{array}$ \\
\hline
\end{tabular}
the dual form of LP 1 which is formally stated as LP 2 .

\footnotetext{
${ }^{4} \mathrm{An}$ additive form of approximation, called $\epsilon$-minimax or $\epsilon$-equilibrium is also common in the literature; however, we emphasize that all of our approximation factors are multiplicative.

${ }^{5}$ We remark that our results in this section have no assumption on the structure of the defender's strategy set $\mathcal{S}^{D}$ and can be adopted to other models of security games (i.e., not necessarily spatio-temporal) with similar payoff structures.
} 
Note that LP 2 also has exponentially many variables. However, the simplicity of valid pure strategies of the attacker helps us in obtaining an equivalent LP with only polynomially many variables. To elaborate more on this, first note that the first 3 constraints of LP 2 ensure only that $y$ is a valid mixed strategy of the attacker. That is, y must be a linear combination of the strategies in $\mathcal{S}^{A}$. To do this, LP 2 includes a variable $p_{a}$ for every strategy a $\in \mathcal{S}^{A}$, indicating the probability of playing strategy a. Since the attacker has $\left(\begin{array}{l}N \\ Q\end{array}\right)$ different pure strategies, this leads to an exponential (in $Q)$ number of variables. However, observe that these variables are only used to ensure validity of vector $y$ (i.e., they do not appear in the other constraints). To simplify LP 2 and get rid of the exponential number of variables, we show an alternative way that ensures validity of $\mathbf{y}$ via only polynomially many variables. Note that any valid mixed strategy $\mathbf{y}$ of the attacker should satisfy the following two conditions:

(1) For any $i \in[N], 0 \leq y_{i} \leq 1$,

(2) $\sum_{i \in[N]} y_{i}=Q$.

In Lemma 2 we show that these conditions are also sufficient to ensure $\mathbf{y}$ is a valid strategy.

Lemma 2. Let $\mathbf{y}=\left(y_{1}, \ldots, y_{N}\right)$ be a vector satisfying $\sum_{i \in[N]} y_{i}=Q$ and $0 \leq y_{i} \leq 1$ for any $i \in[N]$. There exists a mixed strategy for the attacker that provides marginal attacking probability $\mathbf{y}$.

In the interest of space, we defer the proof of Lemma 2 to the full-version of the paper. Roughly speaking, given a vector $y$ that satisfies the aforementioned constraints, we iteratively extract a pure strategy $\mathbf{s} \in \mathcal{S}^{A}$ with an assigned probability $p$ and update $\mathbf{y}$ to be $\mathbf{y}-p \mathbf{s}$. The challenge is to make sure that there are at least $Q$ non-zero entries in y at every step and that the probabilities that we assign to the strategies does not exceed 1 when $y$ becomes zero.

As a result of Lemma 2, we can simplify LP 2 to obtain an equivalent LP - formally given as LP 3 - with only polynomially many variables.

$$
\begin{array}{rlr}
\max & \mathbf{y} \cdot \mathbf{w}-c, & \\
\text { s.t. } & 0 \leq y_{i} \leq 1 & \forall i \in[N], \\
& \sum_{i \in[N]} y_{i}=Q, & \\
& h_{i}=\left(w_{i}-c_{i}\right) y_{i} & \forall i \in[N], \\
& \mathbf{h} \cdot \mathbf{d} \leq c & \forall \mathbf{d} \in \mathcal{S}^{D} .
\end{array}
$$

LP 3. A simplified version of LP 2 with polynomially many variables. Variables are $y_{i}$ (probability of attacking target $i), h_{i}$, and $c$ (the defender's coverage).

Despite having a polynomial number of variables, the number of constraints in LP 3 is still exponentially large. Nevertheless, we can use the ellipsoid method to solve LP 3 in polynomial time if we have access to a polynomial time separation oracle that for any assignment to the variables of LP 3 either asserts that the assignment does not violate any of the constraints or otherwise returns a violated constraint. Verifying that the first three constraint sets in LP 3 are not violated can easily be done in polynomial time since there are only $2 N+1$ such constraints. The main challenge is to confirm that no constraint of the fourth type is violated. This can be achieved by solving the defender's best response problem, which we formally define as follows.

Definition 3 (Defender's best response problem). For a given weight vector $\mathbf{h} \in \mathbb{R}_{+}^{N}$, the defender's best response problem,

$$
\operatorname{DBR}(\mathbf{h}):=\arg \max _{\mathbf{s} \in \mathcal{S}^{D}}[\mathbf{h} \cdot \mathbf{s}]
$$

is to find a pure strategy that covers the maximum possible weight. Moreover, we denote by $\mathrm{C}(\mathbf{h}):=\max _{\mathbf{s} \in \mathcal{S}^{D}}[\mathbf{h} \cdot \mathbf{s}]$ the best possible coverage for the defender, i.e., $\mathrm{C}(\mathbf{h})=\mathbf{h} \cdot \operatorname{DBR}(\mathbf{h})$. 
Observation 4. The separation oracle of LP 3 can be solved in polynomial time if DBR can be solved in polynomial time.

It does not immediately follow that a solution for the dual LP implies a solution for the primal LP since the primal LP has exponentially many variables and even constructing a solution may require exponential time. However, it has been proven that in such cases, there always exists a primal solution with polynomially many non-zero variables and that a separation oracle for the dual LP along with the ellipsoid method obtains such a solution (see e.g. [1]). So far, it was shown that if the DBR problem can be solved in polynomial time, the actual game can also be solved in polynomial time. The DBR problem, however, is generally NP-hard even for restricted cases where $T=1$. However, we show that, roughly, any approximation of the DBR problem leads to an approximation of the actual game with the same approx factor. We start by defining approximate DBR.

Definition 5 (Approximate DBR). For any $\alpha \leq 1$ and $\beta \geq 1$, and any given weight vector $\mathbf{h} \in \mathbb{R}_{+}^{N}$, a pure strategy $\mathbf{s}$ of the defender is an $(\alpha, \beta)$-approximation of the DBR problem, if it uses up to $\beta K$ patrols and

$$
\mathbf{h} \cdot \mathbf{s} \geq \alpha[\mathbf{h} \cdot \operatorname{DBR}(\mathbf{h})] .
$$

Theorem 6 implies that both approximation factors $(\alpha$ and $\beta$ ) can be preserved in polynomial time for the actual game. The proof is technical and we defer it to Section 6.

Theorem 6. Having access to an oracle that provides a $(\alpha, \beta)$-approximation of the defender's best response problem, one can achieve in polynomial time, an $\alpha$-approximate maximin strategy of the defender that uses up to $\beta K$ patrols.

\section{APPROXIMATING DEFENDER'S BEST RESPONSE}

We showed that even an approximate solution for the defender's best response (DBR) problem leads to an approximation of the actual game. As such, in this section, we focus on the DBR problem and give a general approach for solving or approximating it.

Recall that in the DBR problem, given a weight vector $\mathbf{h} \in \mathbb{R}_{+}^{N}$, the solution is a defender's pure strategy $\mathbf{s} \in \mathcal{S}$ that maximizes $\mathbf{h} \cdot \mathbf{s}$. In other words, the solution is a pure strategy of the defender that maximizes the total weight of protected targets.

The general approach is to relax the integrality assumption of patrol paths. Roughly speaking, we allow the patrols to be partitioned into fractions with each fraction moving independently. For example, 0.6 fraction of a patrol might pass through a patrol path and the remaining 0.4 fraction through another one. This relaxation allows us to solve the problem optimally via an LP. We then analyse the integrality gap of this LP using different rounding techniques.

We first define a directed graph $\mathcal{T}\left(V_{\mathcal{T}}, E_{\mathcal{T}}\right)$, which we refer to as the temporal graph, in such a way that any patrol path maps to a path in $\mathcal{T}$. Also any fractional patrol path can be represented as a flow of size 1 in this graph. We use this representation to write the Linear Program 4 which finds a fractional solution to the defender's best response problem. Recall that the game is played on graph $G=(V, E)$ where the defender's patrols and the targets can be positioned on the vertices of $G$. Also, recall that the temporal domain has $T$ discretized time points.

Definition 7 (Temporal graph). Given the base graph $G=(V, E)$ on which the game is played, the temporal graph $\mathcal{T}=\left(V_{\mathcal{T}}, E_{\mathcal{T}}\right)$ is defined as follows.

- The vertex set of $\mathcal{T}$ is composed of $T$ copies $V_{1}, \ldots, V_{T}$ of $V$. For any $v \in V$, we denote by $v^{(t)}$ its equivalent vertex in $V_{t}$.

- For any edge $\{u, v\} \in E$ and for any time point $t \in[T-1]$, the edge set $E_{\mathcal{T}}$ of $\mathcal{T}$ contains two directed edges: one from $u^{(t)}$ to $v^{(t+1)}$ and one from $v^{(t)}$ to $u^{(t+1)}$. 
Before proceeding, we need a few definitions over the temporal graph. For any target $a \in[N]$ in time $t_{a}$ and at vertex $\ell_{a}$, we denote by $\operatorname{cover}(a):=\left\{v^{\left(t_{a}\right)}: v^{\left(t_{a}\right)} \in V_{t_{a}}, \operatorname{dist}_{G}\left(v, \ell_{a}\right) \leq R\right\}$ the set of all vertices in $V_{t_{a}}$ from which $a$ is protected in the base graph. Moreover, for any $v \in V_{\mathcal{T}}$, sets $A^{+}(v)$ and $A^{-}(v)$ respectively denote incoming and outgoing edges of $v$.

It is not hard to imagine a one-to-one mapping between the set of all possible patrol paths and paths of length $T$ in $\mathcal{T}$. Note that a path $P$ of length $T$ in $\mathcal{T}$ must start from a vertex in $V_{1}$ and end at a vertex in $V_{T}$ since there are no backward edges. The equivalent patrol path in the base graph is a path of length $T$ that traverses the vertices equivalent to that of $P$ in $G$ in the same order. Throughout the paper, we may slightly abuse the notation and refer to such paths in $\mathcal{T}$ as patrol paths.

Any pure strategy of the defender can be represented by $K$ patrol paths in graph $\mathcal{T}$. To be able to solve the DBR problem in polynomial time, we relax the integrality assumption on the patrol paths. More precisely, we assume that any network flow of size $K$ that starts from the vertices in $V_{1}$ and ends at the vertices in $V_{T}$ is a valid pure strategy. We then write a linear program - which is formally given as LP $4-$ to find a relaxed pure strategy that maximizes the total coverage.

\begin{tabular}{|rlr|}
\hline $\max$ & $\mathbf{p} \cdot \mathbf{h}$ & \\
s.t. & $0 \leq f_{v}$ & $\forall v, t: t \in[T], v \in V_{t}$ \\
& $\sum_{v \in V_{t}} f_{v}=K$ & $\forall t: t \in[T]$ \\
& $\sum_{e \in A^{-}(v)} f_{e}=f_{v}$ & $\forall t, v: t \in[T-1], v \in V_{t}$ \\
& $\sum_{e \in A^{+}(v)} f_{e}=f_{v}$ & $\forall t, v: t \in[2, T], v \in V_{t}$ \\
& $p_{i} \leq \sum_{v \in \operatorname{cover}(i)} f_{v}$ & $\forall i \in[N]$ \\
& $p_{i} \leq 1$ & $\forall i \in[N]$ \\
LP 4. LP relaxation of the DBR problem. Variables are $p_{i}$ (protection of target $i$ ) and $f_{a}$ (amount of flow \\
passing through vertex or edge $a$ ). Find the informal description of the LP in the text.
\end{tabular}

Description of LP 4. Variable $f_{a}$ denotes the amount of flow passing through $a$ where $a$ may be either an edge or a vertex in graph $\mathcal{T}$. The first four constraints are standard network flow constraints to ensure we get a valid flow of size $K$. To maximize the coverage, for any target $i \in[N]$ we use a variable $p_{i}$ that indicates the total coverage of target $i$ in the solution. More precisely, 1 indicates that the target is fully covered and 0 indicates that the target is not covered by any patrol. Note that generally, the amount of flow around a target might be more than 1 , to prevent double coverage in such scenarios, we add a constraint to ensure $p_{i}$ never exceeds 1 . The objective function, clearly, is to maximize the total coverage which is basically $\mathbf{p} \cdot \mathbf{h}$.

The rest of this section is mainly focused on different rounding methods that help us achieve approximation algorithms for the integral DBR problem.

\subsection{One Dimensional Domains}

An important class of security games is played on one-dimensional domains, that is the patrols could move over a discretized line (see Figure 1). This model has been considered several times in the literature because of its numerous real-world applications. A polynomial time algorithm (though, under the assumption that the attacker attacks only once) was previously given by [3]. In this section, we show how by a dependent rounding technique, one can solve LP 4 to obtain an exact solution for the DBR problem in one-dimensional domains and solve the actual game. We note that a similar DBR problem has been considered in [19] where the authors give a $1-1 / e$ approximation for it, using submodular maximization algorithms. Combining this approximation 
with Theorem 6 one can achieve a $1-1 / e$ approximation of the actual game. Our focus in this section is to show how the DBR problem could be optimally solved by rounding LP 4 .

We can model the one-dimensional space via a path of length $M$. However, this limits the patrols to only move to an adjacent vertex in after each time point. The original model is more general than this, given a speed limit $\Delta$, each patrol is able to move to any other location with distance at most $\Delta$. To consider this, we construct the base graph $G_{1}=\left(V_{1}, E_{1}\right)$ as follows: The vertex set $V_{1}$ contains $M$ vertices $\left\{v_{1}, v_{2}, \ldots, v_{M}\right\}$, and two vertices $v_{i}$ and $v_{j}$ are connected to each other iff $|i-j| \leq \Delta$. Intuitively, we add edges between any two locations (i.e., vertices) that are accessible from each other in one time step. Throughout the section, we say a vertex $v_{i}$ is above $v_{j}$ or vertex $v_{j}$ is below vertex $v_{i}$, if $i>j$.

Consider the solution (f, p) of LP 4 for graph $G_{1}$. Recall that $\mathcal{T}=\left(V_{\mathcal{T}}, E_{\mathcal{T}}\right)$ denotes the temporal graph of $G_{1}$ and that $f_{v}$ denotes the amount of flow passing through a vertex $v \in V_{\mathcal{T}}$. The intuition behind our rounding algorithm, as illustrated in Figure 3, is as follows: (1) Choose a fractional number $\sigma$ uniformly at random from $[0,1]$. (2) For any time point $t$, and for any $k \in[K]$, put the $k$ th patrol at the top-most vertex in time $t$ where the amount of flow passing through the vertices below it is less than $k+\sigma$. To prove that this rounding technique works we need to prove two things: That the patrol paths are valid (i.e., the Fig. 3. Intuition of Algorithm 1.1 . and that it provides the same coverage. Roughly speaking, the validity of patrol paths is proved by the structure of the flows. The amount of coverage is also preserved since, we show, this rounding method minimizes double coverage.

\begin{tabular}{l}
\hline Algorithm 1 Rounding technique for the fractional solution of LP 4 in the one-dimensional case. \\
1: Choose a fractional number $\sigma$ in range $[0,1]$ u.a.r. $\quad \triangleright$ Remark: any choice of $\sigma$ works. \\
2: For any $k \in[K]$ and any $t \in[T]$, let $\mathrm{P}(k, t):=\max \left\{i: \sum_{j=1}^{i-1} f_{v_{j}^{(t)}}<k+\sigma\right\}$ be the top-most \\
vertex at time $t$ that the amount of flow passing through the vertices below it is less than $k+\sigma$. \\
3: Return $\left\{\left(v_{\mathrm{P}(k, 1)}, \ldots, v_{\mathrm{P}(k, T)}\right)\right\}_{k \in[K]} \quad \triangleright$ Lemma 8 shows this is a valid set of patrol paths.
\end{tabular}

Lemma 8. The solution of Algorithm 1 is a valid set of patrol paths. That is, for any $k \in[K]$ and any $t \in[T-1]$, we have $|p(k, t)-P(k, t+1)| \leq \Delta$.

Proof. We first assume that the path assigned to patrol $k \in[K]$ violates the condition at time $t \in[T-1]$, then we obtain a contradiction. Let $a:=\mathrm{P}(k, t)$ and $b:=\mathrm{P}(k, t+1)$. This violation means that due to the patrols' limited speed, it is not possible for them to move from vertex $v_{a}$ to $v_{b}$ at one unit of time. More formally, $|a-b|>\Delta$. Therefore, either $b>a+\Delta$ or $a>b+\Delta$.

We first consider the case where $a>b+\Delta$. By Line 2 of Algorithm 1 ,

$$
\sum_{j=1}^{a-1} f_{v_{j}^{(t)}}<k+\sigma, \quad \text { and } \quad \sum_{j=1}^{b} f_{v_{j}^{(t+1)}} \geq k+\sigma ;
$$

therefore,

$$
\sum_{j=1}^{a-1} f_{v_{j}^{(t)}}<\sum_{j=1}^{b} f_{v_{j}^{(t+1)}}
$$

Let $A$ and $B$ respectively denote the set of vertices $\left\{v_{1}^{(t)}, \ldots, v_{a-1}^{(t)}\right\}$ and $\left\{v_{1}^{(t+1)}, \ldots, v_{b}^{(t+1)}\right\}$. Inequality 4 implies that the overall amount of flow passing through vertices in set $A$ is less than that of set $B$. However, by the structure of graph $\mathcal{T}$ and the assumption $a>b+\Delta$, all the incoming edges to vertices in set $B$ are from vertices in set $A$. Hence, any flow passing though $B$ comes from the 
vertices in $A$. This implies that the amount of flow passing through vertices in $A$ cannot be less than the amount of flow passing through $B$ which contradicts Inequality 4.

The case of $b>a+\Delta$ also follows from a similar argument. By Line 2 of Algorithm 1 ,

$$
\sum_{j=1}^{a} f_{v_{j}^{(t)}} \geq k+\sigma, \quad \text { and } \quad \sum_{j=1}^{b-1} f_{v_{j}^{(t+1)}}<k+\sigma ;
$$

therefore,

$$
\sum_{j=1}^{b-1} f_{v_{j}^{(t+1)}}<\sum_{j=1}^{a} f_{v_{j}^{(t)}} .
$$

Let $A^{\prime}=\left\{v_{1}^{(t)}, \ldots, v_{a}^{(t)}\right\}$ and let $B^{\prime}=\left\{v_{1}^{(t+1)}, \ldots, v_{b-1}^{(t+1)}\right\}$. Inequality 5 implies that the overall amount of flow passing through vertices in set $B^{\prime}$ is less than that of set $A^{\prime}$. However, by the structure of graph $\mathcal{T}$ and the assumption $b>a+\Delta$, all the outgoing edges of the vertices in $A^{\prime}$ are to a vertex in $B^{\prime}$. Hence, any flow passing though $A^{\prime}$ also passes through $B^{\prime}$; which contradicts Inequality 4.

In Lemma 9 we prove that the expected coverage of the integral solution given by Algorithm 1 is equal to that of the fractional one. Let $\mathrm{OPT}_{4}$ denote the coverage of the fractional solution given by LP 4. Also let vector $\mathbf{x}$ be the marginal coverage probability of the pure strategy that is obtained by Algorithm 1. Note that $\mathbf{x}$ is a random variable due to the randomness in the rounding algorithm.

Lemma 9. Algorithm 1 provides the same coverage as $L P 4$ in expectation, that is $\mathbb{E}[\mathbf{h} \cdot \mathbf{x}]=O P T_{4}$.

Proof. Since $\mathrm{OPT}_{4}=\mathbf{p} \cdot \mathbf{h}$, by linearity of expectation it suffices to prove that for any $a \in[N]$, we have $\mathbb{E}\left[x_{a}\right] \geq p_{a}$ holds. For an arbitrary target $a$, let $i$ and $j$ respectively denote the smallest and the greatest number in $[M]$ where $v_{i}^{\left(t_{a}\right)}, v_{j}^{\left(t_{a}\right)} \in \operatorname{cover}(a)$. Note that for any $k \in[i, j]$, vertex $v_{k}^{\left(t_{a}\right)}$ is also in $\operatorname{cover}(a)$. We prove our claim using the fact that $x_{a}=1$ iff there exists a $k \in[M]$, where $\mathrm{P}\left(k, t_{a}\right) \in[i, j]$. Let $l_{a}:=\sum_{k \in[i-1]} f_{v_{k}^{(t)}}$ and $r_{a}:=\sum_{k \in[j]} f_{v_{k}^{(t)}}$. Note that $p_{a} \leq r_{a}-l_{a}$. We consider 3 different cases based on values of $l_{a}$ and $r_{a}$.

- $\left\lceil l_{a}\right\rceil=\left\lceil r_{a}\right\rceil$ : let $k:=\left\lceil l_{a}\right\rceil$. In this case, $p\left(k, t_{a}\right) \in[i, j]$ iff $l_{a}-\left\lfloor l_{a}\right\rfloor<\sigma \leq r_{a}-\left\lfloor r_{a}\right\rfloor$. Therefore, $\mathbb{E}\left[x_{a}\right]=r_{a}-l_{a}$, and $\mathbb{E}\left[x_{a}\right] \geq p_{a}$.

- $\left\lceil r_{a}\right\rceil-\left\lceil l_{a}\right\rceil=1$ : let $k_{1}:=\left\lceil l_{a}\right\rceil$ and $k_{2}:=\left\lceil r_{a}\right\rceil$. In this case, $l_{a}-\left\lfloor l_{a}\right\rfloor<\sigma \leq 1$ iff $p\left(k_{1}, t_{a}\right) \in[i, j]$. Also, $0 \leq \sigma \leq r_{a}-\left\lfloor r_{a}\right\rfloor$ iff $p\left(k_{2}, t_{a}\right) \in[i, j]$. Therefore, $\mathbb{E}\left[x_{a}\right]=\min \left(1, r_{a}-l_{a}\right)$ and $\mathbb{E}\left[x_{a}\right] \geq p_{a}$.

- $\left\lceil r_{a}\right\rceil-\left\lceil l_{a}\right\rceil>1$ : in this case, $p\left(\left\lceil r_{a}\right\rceil+1, t_{a}\right) \in[i, j]$ always holds, therefore $1=p_{i}=\mathbb{E}\left[x_{a}\right]$.

Three mentioned cases covers all the possiblities for the relation between $l_{a}$ and $r_{a}$, and $p_{a} \leq \mathbb{E}\left[x_{a}\right]$ holds in three of them. Thus for any $a \in[N], p_{a} \leq \mathbb{E}\left[x_{a}\right]$ and as a result, $\mathrm{OPT}_{4}=\mathbb{E}[h \cdot x]$.

Note that, any integral solution is also a valid fractional solution for LP 4 . Since LP 4 finds the solution with maximum coverage, no integral solution can provide a higher coverage than the solution of LP 4. Implying that in order for the expected value of $\mathbb{E}[\mathbf{h} \cdot \mathbf{x}]$ to be the same as $\mathrm{OPT}_{4}$, for every choice of $\sigma$, the integral solution should provide the same coverage as LP 4.

Corollary 10 (of Lemma 9). For any choice of $\sigma$, Algorithm 1 provides the same coverage as LP 4.

To summarize, recall that the input to the DBR problem is a weight vector $\mathbf{h} \in \mathbb{R}_{+}^{N}$. We first find a an optimal fractional solution using LP 4; then round it using Algorithm 1 to obtain an integral solution. The output of this algorithm is $k$ patrol paths, each assigned to a patrol. In Lemma 8, we prove that these paths do not violate the speed limit and show in Corollary 10 that for any choice of $\sigma$, this algorithm provides the same coverage as LP 4. Meaning that the integrality gap of LP 4, is 1 for the one dimensional case (i.e., over graph $G_{1}$ ) and that we can solve the DBR problem optimally in polynomial time. 
Theorem 11. There exists an algorithm that solves the defender's best response (DBR) problem optimally for the one-dimensional case (i.e., over graph $G_{1}$ ) in polynomial time.

Combined with Theorem 6 (for $\alpha=\beta=1$ ), this implies that the optimal minimax strategy of the defender for the one-dimensional case can be obtained in polynomial time.

Corollary 12 (of Theorem 12). There exists a polynomial time algorithm to find the defender's optimal minimax strategy for the one-dimensional case (i.e., over graph $G_{1}$ ) in polynomial time.

Generalization to cycles. Assume that the base graph is a cycle, or even a pseudo-cycle where each node of the cycle also has edges to its $\Delta$ nearby vertices. We argue here that essentially the same rounding procedure can be slightly modified to obtain an exact solution. For space limits, we do not provide a detailed proof and only describe the high level ideas behind the solution. Roughly speaking, the challenge compared to path graph, is that the patrols may need to rotate around the cycle over time. That is, the same approach of starting from the bottom-most vertex and putting a patrol at the first vertex that contains a flow of size $\sigma+k$ below it does not work for cycles. Instead, for each time point $t$, we fix a different vertex $v_{t}$ and start the same procedure from this vertex. To fix these vertices, we first decompose the flow of LP 4 into $K$ flows of size 1 in such a way that the $i$ th flow is sandwiched between the $i-1$ th and the $i+1$ th flows. ${ }^{6}$ Next, it suffices to pick two flows, say the first and the second, and for each time point $t$, fix a vertex $v_{t}$ that is sandwiched between the first and the second flow.

\subsection{General Graphs}

The goal of this section is to show that one can achieve, in polynomial time, a near-optimal solution of defender's best response problem by allowing the defender to have a slightly greater number of patrols. Theorem 15 formalizes the main result of this section. To prove this theorem we first give a rounding technique in Algorithm 2 for converting the fractional solution given by LP 4 to an integral one. Note that the integral solution is an integral pure strategy of the defender. We, then prove that solving the LP combined with this rounding technique gives us the approximation mentioned in Theorem 15.

Given a solution of LP 4 and an $\epsilon>0$, Algorithm 2 outputs an integral pure strategy of the defender that uses up to $\lceil\ln (1 / \epsilon)\rceil \cdot K$ patrols. Graph $\mathcal{T}=\left(V_{\mathcal{T}}, E_{\mathcal{T}}\right)$ is the temporal graph of graph $G$. Recall that any solution of LP 4 is a flow of size $K$ from $V_{1}$ to $V_{T}$ in graph $\mathcal{T}$. Let $F$ denote this flow. Algorithm 2, first decomposes $F$ into a set of paths denoted by $P$. (The definition of flow decomposition is given in Definition 13.) There exists a simple greedy algorithm that decomposes $F$ into polynomially many paths. It is possible to iteratively choose a flow path and remove it from $F$ such that the remaining flow of at least one edge decreases to 0 after each iteration. Therefore, the greedy algorithm returns a set $P$ where $|P|$ is polynomial in the size of $E_{\mathcal{T}}$. In the next step, Algorithm 2, partitions $P$ into $K$ subsets denoted by $P_{1} \ldots P_{K}$ where for any $i \in K, \sum_{p \in P_{i}} f_{p}=1$ Note that, paths in $P$ can be decomposed to some fractions, thus it is possible to find such a partitioning in $O(|P|)$ time. For any $i \in K$, Algorithm 2 independently at random chooses $\lceil\ln (1 / \epsilon)\rceil$ patrol paths from each $P_{i}$. The randomness is in a way that the probability of choosing any path is equal to the flow assigned to that. In Lemma 14, we prove that in expectation this algorithm gives a $(1-\epsilon,\lceil\ln (1 / \epsilon)\rceil)$-approximation of for defender's best response problem.

Definition 13 (Flow decomposition). Flow $F$ denotes a flow on graph $\mathcal{T}$ from $V_{1}$ to $V_{T}$. Let $f_{e}$ be the amount of flow that passes through any edge $e \in E_{\mathcal{T}}$ in $F$. Decomposition of $F$ is a set $P$ of paths from $V_{1}$ to $V_{T}$ with a nonzero amount of flow assigned to any one. For any path $p \in P$,

\footnotetext{
${ }^{6}$ This requires a postprocessing step on the solution of LP 4 to remove its crossing edges.
} 
denote by $f_{p}$ the amount of flow that is assigned to $p$. Let $P_{e}=\{p: p \in P, e \in p\}$. For any valid flow decomposition of $F, \sum_{p \in P_{e}} f_{p}=f_{e}$ and $|P|$ is polynomial in the size of $E_{\mathcal{T}}$.

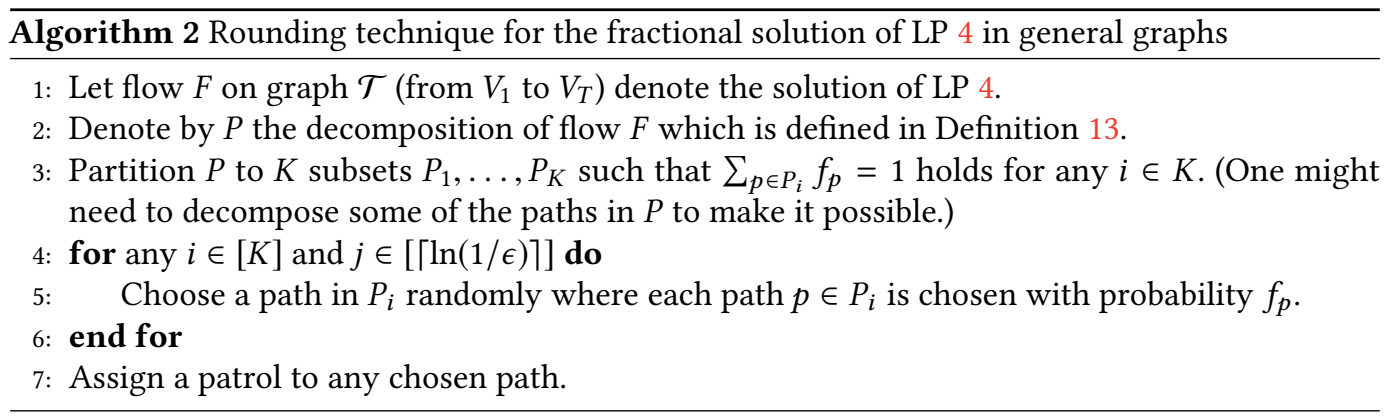

The following lemma shows that Algorithm 2 indeed provides a $(1-\epsilon)$-approximation. Due to space limit, the proof of this lemma is left to the full-version of the paper.

Lemma 14. For any given weight vector $\mathbf{h} \in \mathbb{R}_{+}^{N}$ and any constant $\epsilon>0$, there exists an approximate defender best response $(A D B R)$ algorithm that using $K^{\prime}=\lceil\ln (1 / \epsilon)\rceil \cdot K$ patrols, finds a random strategy $\mathrm{s} \in \mathcal{S}_{K^{\prime}}$ in polynomial time, where

$$
\mathbb{E}[\mathbf{h} \cdot \mathbf{s}] \geq(1-\epsilon) \cdot C_{K}(\mathbf{h}) .
$$

By combining this with Theorem 6 we get the following results.

Theorem 15. For any $\epsilon>0$, there exists an algorithm, that in time $\operatorname{poly}(\eta, \log (1 / \epsilon))$ finds in expectation a $(1-\epsilon)$-approximate minimax strategy of the defender, using up to $\lceil\ln (1 / \epsilon)\rceil \cdot K$ patrols.

We remark there is also another way to obtain the result above using a greedy algorithm that provides the same approximation factor exactly (i.e., not in expectation).

\section{PRESERVING THE APPROXIMATION FACTOR}

The goal of this section is to show that any approximation (of a certain type) for the defender's best response problem, even when we relax the assumption that the defender has to use at most $K$ patrols, leads to an approximate minimax strategy.

Since in this section we allow the defender to use more than $K$ patrols, we need to slightly modify some notations to clearly state the number of patrols that are used in different parts of the algorithm. Recall that we denote by $\mathcal{S}^{D}$ the set of all pure strategies of the defender. Here, we use $\mathcal{S}_{j}^{D}$ to denote the set of all pure strategies of the defender that use up to $j$ patrols; meaning that $\mathcal{S}_{K}^{D}=\mathcal{S}^{D}$. Moreover, we generalize Definition 3 and denote by $\operatorname{DBR}_{j}(\mathbf{h}):=\arg \max _{\mathbf{s} \in \mathcal{S}_{j}^{D}}[\mathbf{h} \cdot \mathbf{s}]$ the defender's best response to weight vector $\mathbf{h}$ when she is able to use $j$ patrols. Similarly, we use $\mathrm{C}_{j}(\mathbf{h}):=\max _{\mathbf{s} \in \mathcal{S}_{j}^{D}}[\mathbf{h} \cdot \mathbf{s}]$ to denote the coverage of defender's best response that uses up to $j$ patrols.

We are now ready to formally define approximate DBR and state the main theorem of this section.

Definition 16 (Approximate DBR). For any $\alpha \leq 1$ and $\beta \geq 1$, we call an oracle $O: \mathbb{R}_{+}^{N} \rightarrow \mathcal{S}_{\beta K}^{D}$, an $(\alpha, \beta)$-approximation of the defender's best response problem, if for any $\mathbf{h} \in \mathbb{R}_{+}^{N}$, we have

$$
\mathbf{h} \cdot O(\mathbf{h}) \geq \alpha \cdot \mathrm{C}_{K}(\mathbf{h}) .
$$

To simplify notations, when $\beta=1$, we simply use $\alpha$-approximation instead of $(\alpha, 1)$-approximation. 
Theorem 6 Having access to an $(\alpha, \beta)$-approximation oracle for the defender's best response problem, one can achieve in polynomial time, an $\alpha$-approximate maximin strategy of the defender that uses up to $\beta K$ patrols.

We cannot directly use an approximate best response as the separating oracle of LP 3 since the found strategies can use more than $K$ patrols by definition, whereas, LP 3 constraints the defender to use at most $K$ patrols. More precisely, the violating constraint that is implied by the oracle might not exist in LP 3. To overcome this, we write a modified version of LP 3, which first allows the defender to use up to $K^{\prime}:=\beta K$ patrols, and second, converts the maximization problem to a feasibility problem. Here we restate LP 3 and state its modified version as LP 5 .

$$
\begin{aligned}
& \max \mathbf{y} \cdot \mathbf{w}-c, \\
& \text { s.t. } 0 \leq y_{i} \quad \forall i \in[N] \text {, } \\
& \sum_{i \in[N]} y_{i}=Q \text {, } \\
& h_{i}=\left(w_{i}-c_{i}\right) y_{i} \quad \forall i \in[N] \text {, } \\
& \mathbf{h} \cdot \mathbf{s} \leq c \quad \forall \mathbf{s} \in \mathcal{S}_{K}^{D} \text {. }
\end{aligned}
$$

LP 3 (restated): Variables are $y_{i}, h_{i}$, and $c$.

LP 5. Variables are $y_{i}, h_{i}$, and $c$.

We need a few notations before proceeding. Let us denote by $\mathrm{OPT}_{3}$ the optimum value of LP 3 . We also denote by $\operatorname{FEAS}_{5}\left(b^{\prime}\right) \in\{0,1\}$ whether LP 5 has any feasible solution by setting the constant value $b$ to be $b^{\prime}$ or not (1 indicates feasibility and 0 indicates infeasibility). LP 5 does not have a direct objective function, but we denote by $\mathrm{OPT}_{5}:=\max \left\{b^{\prime}: \operatorname{FEAS}_{5}\left(b^{\prime}\right)=1\right\}$ the maximum possible value of $b$ for which a feasible solution exists. Notice that $\mathrm{OPT}_{3}$ and $\mathrm{OPT}_{5}$ respectively denote the optimal utility of the attacker when the defender can use up to $K$ and $K^{\prime}$ patrols. Therefore, since $K^{\prime} \geq K$, the value of $\mathrm{OPT}_{5}$ cannot be more than $\mathrm{OPT}_{3}$.

Observation 17. $O P T_{5} \leq O P T_{3}$.

We cannot compute either of $\mathrm{OPT}_{3}$ or $\mathrm{OPT}_{5}$ in polynomial time. What we show, however, is that we can approximate $\mathrm{OPT}_{5}$ in polynomial time to obtain a solution that we later argue is sufficiently close to $\mathrm{OPT}_{3}$.

For a fixed $b$, we approximately check the feasibility of LP 5 by using the approximate best response as the separating oracle of ellipsoid method. Let us denote the obtained solution by $\operatorname{AFEAS}(b)$, where $\operatorname{AFEAS}(b)=1$ if the ellipsoid method finds a feasible solution, and $\operatorname{AFEAS}(b)=0$ otherwise. Obviously, since the ellipsoid method uses an approximate separating oracle, it does not always return the correct solution, i.e., $\operatorname{AFEAS}(b)$ is not necessarily equal to $\operatorname{FEAS}(b)$. However, it is still possible to have the following deduction about the value of $\operatorname{AFEAS}(b)$.

Claim 6.1. If $\operatorname{AFEAS}(b)=0$, then $F E A S(b)=0$.

We do not argue here whether if $\operatorname{AFEAS}(b)=1$ implies $\operatorname{AFEAS}\left(b^{\prime}\right)=1$ for any $b^{\prime}<b$ or not (which might or might not be the case). Regardless, we can binary search in range $[0, W]$ to find at least a locally maximum value $b^{\star}$ where $\operatorname{AFEAS}\left(b^{\star}\right)=1$ and for some $\epsilon^{\prime}>0$ and any $\delta$ with $0<\delta<\epsilon^{\prime}, \operatorname{AFEAS}\left(b^{\star}+\delta\right)=0$. Claim 6.2 formalizes our claim and emphasizes that it takes only polynomial time to find $b^{\star}$. The following figure illustrates the values of $\operatorname{FEAS}(b)$ and $\operatorname{AFEAS}(b)$ based on $b$.

Claim 6.2. There exists a polynomial time algorithm that returns $b^{\star}$ where AFEAS $\left(b^{\star}\right)=1$ and for some $\epsilon^{\prime}>0$ and any $\delta$ with $0<\delta<\epsilon^{\prime}$, AFEAS $\left(b^{\star}+\delta\right)=0$. 

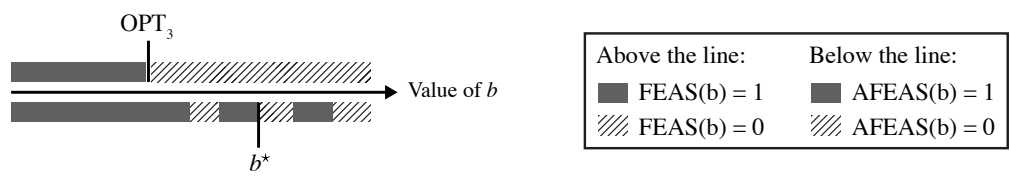

We now prove that $b^{\star}$ is indeed close to $\mathrm{OPT}_{5}$, and therefore, $\mathrm{OPT}_{3}$.

Claim 6.3. $b^{\star} \leq O P T_{3}+(1-\alpha)\left(\mathbf{y}^{\star} \cdot \mathbf{w}\right)$.

Recall, again, that $b^{\star}$ and $\mathrm{OPT}_{3}$ specify the attacker's payoff. To analyze the approximation ratio for the defender, we need to give a lower bound for $\left(\Gamma-b^{\star}\right) /\left(\Gamma-\mathrm{OPT}_{3}\right)$.

Claim 6.4. $\left(\Gamma-b^{\star}\right) /\left(\Gamma-O P T_{3}\right) \geq \alpha$.

In the interest of space, proof of Claim 6.4 is deferred to the full-version of the paper.

We are now ready to prove Theorem 6 .

Proof of Theorem 6: Let $O$ be the $(\alpha, \beta)$-aproximate oracle. We first find a locally optimal solution $b^{\star}$ for LP 5 in polynomial time by Claim 6.2 and by using $O$ as the oracle. We know by Claim 6.4 that

$$
\frac{\Gamma-b^{\star}}{\Gamma-\mathrm{OPT}_{3}} \geq \alpha
$$

Note that $\Gamma-b^{\star}$ and $\Gamma-\mathrm{OPT}_{3}$ respective indicate the defender's utility in the approximate solution and the optimal solution, hence, the approximation factor is precisely $\frac{\Gamma-b^{\star}}{\Gamma-\mathrm{OPT}_{3}}$ which is shown to be at least $\alpha$.

\section{HARDNESS RESULTS}

Hardness of defender's best response. We show in this section that the DBR problem is NP-hard to even approximate within a $(1-1 / e+o(1), 1)$ factor. A matching upper bound of $(1-1 / e, 1)$ can be obtained in polynomial time by setting $\epsilon$ to be $1 / e$ in Theorem 15 .

Theorem 18. There is no $(1-1 / e+o(1))$-approximation of the defender's best response problem unless $\mathrm{P}=\mathrm{NP}$.

Reducing the game to defender's best response. We showed in Section 4 that by solving the DBR problem in polynomial time, one can solve the actual game in polynomial time. However, in some cases, the DBR problem turns out to be hard to solve in polynomial time, as it was shown in the previous section. Does this imply that the game is also hard to solve? In this section, we partially answer this in the affirmative. The proof adopts the recent results of [18] to our settings. We defer the proof to the full-version of the paper.

Theorem 19. When $Q=1$, an instance of spatio-temporal security games can be solved in polynomial time if and only if the DBR problem can be solved in polynomial time.

\section{ACKNOWLEDGMENTS}

We thank Alex Slivkins for many useful discussions and his previous collaboration in [3] that started this work.

\section{REFERENCES}

[1] AmirMahdi Ahmadinejad, Sina Dehghani, MohammadTaghi Hajiaghayi, Brendan Lucier, Hamid Mahini, and Saeed Seddighin. 2016. From Duels to Battlefields: Computing Equilibria of Blotto and Other Games.. In AAAI. 376-382. 
[2] Soheil Behnezhad, Sina Dehghani, Mahsa Derakhshan, MohammadTaghi HajiAghayi, and Saeed Seddighin. 2017. Faster and Simpler Algorithm for Optimal Strategies of Blotto Game. In Proceedings of the Thirty-First AAAI Conference on Artificial Intelligence.

[3] Soheil Behnezhad, Mahsa Derakhshan, MohammadTaghi Hajiaghayi, and Aleksandrs Slivkins. 2017. A Polynomial Time Algorithm for Spatio-Temporal Security Games. In Proceedings of the 2017 ACM Conference on Economics and Computation. ACM, 697-714.

[4] Branislav Bošanskỳ, Viliam Lisỳ, Michal Jakob, and Michal Pěchouček. 2011. Computing time-dependent policies for patrolling games with mobile targets. In The 10th International Conference on Autonomous Agents and Multiagent Systems-Volume 3. International Foundation for Autonomous Agents and Multiagent Systems, 989-996.

[5] Matthew Brown, Arunesh Sinha, Aaron Schlenker, and Milind Tambe. 2016. One Size Does Not Fit All: A GameTheoretic Approach for Dynamically and Effectively Screening for Threats. In Proceedings of the Thirtieth AAAI Conference on Artificial Intelligence. 425-431.

[6] Vincent Conitzer and Tuomas Sandholm. 2006. Computing the optimal strategy to commit to. In Proceedings 7th ACM Conference on Electronic Commerce (EC-2006). 82-90.

[7] Bruno Escoffier and Vangelis Th Paschos. 2006. Completeness in approximation classes beyond apx. Theoretical computer science 359, 1-3 (2006), 369-377.

[8] Fei Fang, Albert Xin Jiang, and Milind Tambe. 2013. Optimal patrol strategy for protecting moving targets with multiple mobile resources. In Proceedings of the 2013 international conference on Autonomous agents and multi-agent systems. International Foundation for Autonomous Agents and Multiagent Systems, 957-964.

[9] Fei Fang, Thanh Hong Nguyen, Rob Pickles, Wai Y Lam, Gopalasamy R Clements, Bo An, Amandeep Singh, Milind Tambe, and Andrew Lemieux. 2016. Deploying PAWS: Field Optimization of the Protection Assistant for Wildlife Security.. In AAAI. 3966-3973.

[10] Nicole Immorlica, Adam Tauman Kalai, Brendan Lucier, Ankur Moitra, Andrew Postlewaite, and Moshe Tennenholtz. 2011. Dueling algorithms. In Proceedings of the forty-third annual ACM symposium on Theory of computing. ACM, 215-224.

[11] Manish Jain, Erim Kardes, Christopher Kiekintveld, Fernando Ordónez, and Milind Tambe. 2010. Security Games with Arbitrary Schedules: A Branch and Price Approach.. In AAAI.

[12] Christopher Kiekintveld, Manish Jain, Jason Tsai, James Pita, Fernando Ordóñez, and Milind Tambe. 2009. Computing optimal randomized resource allocations for massive security games. In Proceedings of The 8th International Conference on Autonomous Agents and Multiagent Systems-Volume 1. International Foundation for Autonomous Agents and Multiagent Systems, 689-696.

[13] Dmytro Korzhyk, Vincent Conitzer, and Ronald Parr. 2010. Complexity of Computing Optimal Stackelberg Strategies in Security Resource Allocation Games.. In AAAI.

[14] Joshua Letchford and Vincent Conitzer. 2013. Solving Security Games on Graphs via Marginal Probabilities. In Proceedings of the Twenty-Seventh AAAI Conference on Artificial Intelligence.

[15] James Pita, Manish Jain, Janusz Marecki, Fernando Ordóñez, Christopher Portway, Milind Tambe, Craig Western, Praveen Paruchuri, and Sarit Kraus. 2008. Deployed ARMOR protection: the application of a game theoretic model for security at the Los Angeles International Airport. In Proceedings of the 7th international joint conference on Autonomous agents and multiagent systems: industrial track. International Foundation for Autonomous Agents and Multiagent Systems, 125-132.

[16] Tim Roughgarden. 2010. Computing Equilibria: A Computational Complexity Perspective. Economic Theory 42, 1 (2010), 193-236.

[17] Milind Tambe. 2011. Security and game theory: algorithms, deployed systems, lessons learned. Cambridge University Press.

[18] Haifeng Xu. 2016. The mysteries of security games: Equilibrium computation becomes combinatorial algorithm design. In Proceedings of the 2016 ACM Conference on Economics and Computation. ACM, 497-514.

[19] Haifeng Xu, Fei Fang, Albert Xin Jiang, Vincent Conitzer, Shaddin Dughmi, and Milind Tambe. 2014. Solving Zero-Sum Security Games in Discretized Spatio-Temporal Domains.. In AAAI. Citeseer, 1500-1506.

[20] Zhengyu Yin, Albert Xin Jiang, Milind Tambe, Christopher Kiekintveld, Kevin Leyton-Brown, Tuomas Sandholm, and John P Sullivan. 2012. TRUSTS: Scheduling randomized patrols for fare inspection in transit systems using game theory. AI Magazine 33, 4 (2012), 59. 